\title{
Companied Edge Flow Histogram and Color Histogram to Represent Tracking Objects
}

\author{
Sallama Resen \\ PhD. Student, computer \\ sciences \\ Babylon University \\ Baghdad, Iraq
}

\author{
Hala Bahjat, Ph.D \\ head Information System \\ branch \\ Technology university, \\ Baghdad, Iraq
}

\begin{abstract}
Tracking moving objects from moving platform in videos sequence is a challenging task .object movement and platform movement are sources of variations in scene. Mean Shift Algorithm (MSA) is the common tracking algorithm due simple and efficient procedure. Correct Background Weight Histogram(CBWH)decrease background effect in target representation module .

The main drawbacks in MSA is the ineffective model representation to handle illumination variation and occultation problems . MSA failed to track objects in video containing wide ranges of variations and background motion. In this paper motion information is exploited from edge flow by gradient differential. Histogram for edge flow combined with color histogram called Motion Flow Histograms (MFH).MFH used to represent tracking target between two successive frames. New module target representation reduces the false tracker rate without evident increasing time compare to the classical tracking MSA and $\mathrm{CBWH}$.
\end{abstract}

\section{General Terms}

Moving platform, tracking algorithm

\section{Keywords}

MSA; Edge Flow Histogram; Gradient Differentiation Histogram, CBWH;

\section{INTRODUCTION}

Object tracking is major subject in computer vision applications such as traffic road management, surveillance and auto driver [1,2]. traffic control improved and management for treat the blocking problem . One of the most challenging topics in dynamic sight due changes in the objects shape, size diversity moving object speed relative to other objects cause problems such as occlusions, clutters with background environment. Number of algorithms proposed for visual object tracking algorithm, such as mean-shift, Correct Background Weight Histogram and particle filters[3,4].

Mean Shift Algorithm (MSA) is common due to its simple and efficient procedure with suitable accuracies [5]. Camera or the background assumed static in most existing work in this area. Tracking become hard task when both object and backgrounds are changing. To realize moving objects tracking system, researchers in this area face some difficulties such as how to represent and characterize moving objects efficiently [3]. tracking through video frames when sight including multiple objects with different characterize. objects rear move in the same speed and direction so different moving objects probably holds diverse magnitudes and diverse velocities .
With respect to previous difficulties; the main challenge is chose features that able to represent tracking targets. Flow of movement are very characteristic of objects, so tracker performance can potentially be improved when employ motion information. In case, tracking moving object from a moving platform applications in which the background in the scene move relative to the platform movement. new module apply to represent moving object. Motion occurs in each frames is due to either motion parallax between the camera, object and background[2]. independent motion in the scene related to objects movement. Differentials of parallax flows are concentrated essentially at 3D depth boundaries. independent motions are largest at motion boundaries. The main challenge is the efficient representation model characterize motion have abilities to tracking moving object based on movement displacement and origination. Classical mean shift tracking algorithm described in [6]. Mean shift tracker works by searching in each frame for the location of an image region whose color histogram is closest to the reference color histogram of the target [7]. Features and representation of target model, target candidates and geometric relations between them are the main subject of the MSA [8].In this paper develop framework by integrate displacement, origination of edge flow histogram with appearance changing. it employs gradient differential histograms of edge flow as motion features to initialized object of interest in videos. this paper introduces and evaluates new representation combined with original MSA to tracking moving object in complex environment .

\section{RELATED WORK}

Automatic tracking techniques often involve maintaining a model, which is related to the spatial relationship between features [9].In the literature, some features, such as color, shape, texture, contours and motion i.e., trajectory can be used to track the moving objects in videos .Most of the existing methods assumed the camera or background is largely static [10]. Mean shift Algorithm is estimator of nonparametric density function by computes the nearest mode iteratively [11]. Original mean shift tracking algorithm assumed translation movement only and ignore rotation representation. In practical experience appearance of target will be changed in translation and origination. In [12], an extension was proposed to cope with the origination variation. However, little has been done to extend the tracker for rotational motions [13].

MSA assumed object movement is translational Thus; any kind of local differential of flow effects of object rotation is eliminated .In this paper apply edge flow to extract motion feature and introduce an efficient representation of the gradient differential histogram for estimate displacement and 
origination of moving object. No additional parameter is required and the additional processing time is insignificant.

\section{PROPOSED MODEL} REPRESENTATION

The main idea of proposed representation is that we believe that motion is rich enough to conform tracking moving objects against dynamic backgrounds

\subsection{Edge Flow}

Camera saturation cause large changes in magnitude and not in orientation. This show displacement of moving object when background static .video capture by camera raised on moving platform change in scene causes by two sources object and background .The spatial temporal relationship between moving objects boundary is important to define the relative motion of object to platform movement.

Edge provides much of the available appearance information; Edge Flow tries to capture the displacement and orientations of moving object. Edge Flow treat flow components as independent to defined relative objects movement figure(1)represent Edge Flow in four directions $\mathrm{I}_{\mathrm{xy}}, \mathrm{I}_{\mathrm{yx}}, \mathrm{I}_{\mathrm{xx}}, \mathrm{I}_{\mathrm{yy}}$ respectively.
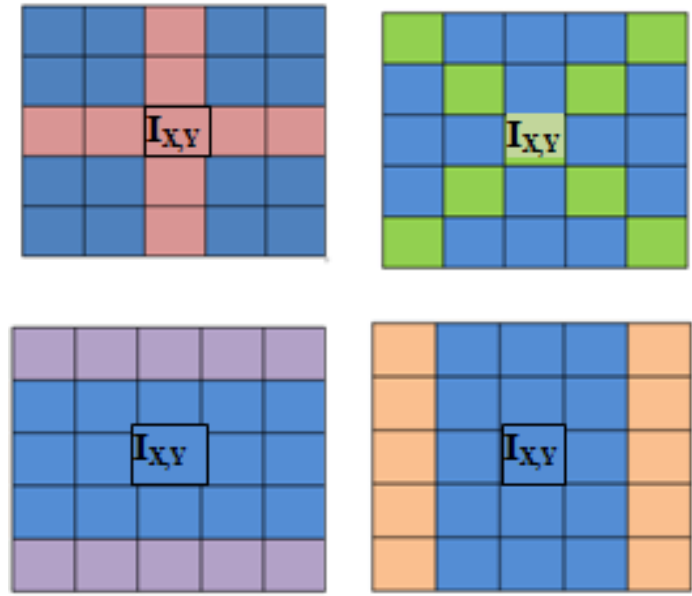

Fig1: four direction gradient difference

Implemented a simple and fast edge flow method based on compute gradient magnitude and orientation for edge. In order to bring information about the magnitude of the intensity changes tack maximum responses from following equation:

$\operatorname{Iyy}(x, y)=(L(x+2, y-2)-L(x-2, y+2)) 2+(L(x+2, y+2)-$ $\mathrm{L}(\mathrm{x}-2, \mathrm{y}-2)) 2$

Фyy $(\mathrm{x}, \mathrm{y})=\arctan (\mathrm{L}(\mathrm{x}+2, \mathrm{y}-2-\mathrm{L}(\mathrm{x}-1, \mathrm{y}+1))+(\mathrm{L}(\mathrm{x}+1, \mathrm{y}+$ 2)-L(x-2, y-1))

$\operatorname{Ixx}(x, y)=(L(x+2, y)-L(x-1, y)) 2+(L(x, y+2)-L(x, y-$ 1)) 2

$\varphi \mathrm{xx}(\mathrm{x}, \mathrm{y})=\arctan (\mathrm{L}(\mathrm{x}, \mathrm{y}+2)-\mathrm{L}(\mathrm{x}, \mathrm{y}-1)) /(\mathrm{L}(\mathrm{x}+2, \mathrm{y})-$ $\mathrm{L}(\mathrm{x}-1, \mathrm{y}))$

$\operatorname{Ixy}(\mathrm{x}, \mathrm{y})=(\mathrm{L}(\mathrm{x}+2, \mathrm{y}+1)-\mathrm{L}(\mathrm{x}-1, \mathrm{y}+1)) 2+(\mathrm{L}(\mathrm{x}+1, \mathrm{y}-1)-$ $\mathrm{L}(\mathrm{x}+1, \mathrm{y}-1)) 2$

$\varphi x y(x, y)=\arctan (L(x+2, y+1)-L(x-1, y+1)) 2+(L(x+1, y-$ 1) $-\mathrm{L}(\mathrm{x}+1, \mathrm{y}-1))$
$\operatorname{Ixy}(\mathrm{x}, \mathrm{y})=(\mathrm{L}(\mathrm{x}+2, \mathrm{y}-2)-\mathrm{L}(\mathrm{x}+2, \mathrm{y}+1)) 2+(\mathrm{L}(\mathrm{x}-2, \mathrm{y}+2)-$ $\mathrm{L}(\mathrm{x}-2, \mathrm{y}-1)) 2$

$\varphi x y(x)=,\arctan \mathrm{L}(\mathrm{x}+2, \mathrm{y}-2)-\mathrm{L}(\mathrm{x}+2, \mathrm{y}+1))+(\mathrm{L}(\mathrm{x}-2, \mathrm{y}+$ $2)-L(x-2, y-1))$

Where L current frame after Gaussian smooth .Maximum gradient responses consider as magnitudes (9) . $\mathrm{M}_{\mathrm{I}, \mathrm{J}}=\operatorname{MAX} \sum\left(\mathrm{I}_{\mathrm{xx}}(\mathrm{x}, \mathrm{y}), \mathrm{I}_{\mathrm{xy}}(\mathrm{x}, \mathrm{y}), \mathrm{I}_{\mathrm{yx}}(\mathrm{x}, \mathrm{y}), \mathrm{I}_{\mathrm{yy}}(\mathrm{x}, \mathrm{y})\right)$

Assign orientation at max response of gardened. Split the interest region up into 5 x 5 square block with regularly spaced sample points inside. Calculate a derivative differential gardened response Ixx , Ixy , Iyx, Iyy for edges. Sum the response over each sub-region for Ixx , Ixy , Iyx, Iyy separately.Magnitudes flow is Maximum response with a square sum difference. Flow origination $\varphi$ calculates for Maximum response.

\subsection{Motion histogram}

Edge flow magnitude histogram provides information that can easily be exploited for tracking. After Edge Flow stage, we wish to calculate the number of pixels having similar Edge Flow value. Set the histogram corresponding to the magnitude of Edge Flow vectors. Local gradient magnitudes and orientations for edge corresponding maximum sum Square difference used as weighted into histograms We call these schemes Motion Flow Histograms (MFH) .

A separate histogram can be built for max responded gradient edge flow. separate histograms are found more discriminate than combined two channels by the winner-takes-all. For motion flow creates histogram with 36 bins.

Compute histogram in magnates and directions for 8 neighboring region. Histogram for color density to interest region combined with Motion Flow Histogram where the intersection returns score for closer histogram joint gradient and color space by equation:

$\mathrm{Q}_{\mathrm{u}}=\mathrm{H}_{\mathrm{m}} \cap \mathrm{H}_{\mathrm{c}}=\sum \min \left(\mathrm{H}_{\mathrm{m}}(\mathrm{i}), \mathrm{H}_{\mathrm{c}}(\mathrm{i})\right)$

Where q Probability Density Function PDF represent target. Mean Shift Tracking Algorithm(MSTA) developed by means of combined color and gradient histogram.

\subsection{Develop MSTA}

In this section we introduce proposed representation to candied target to tracking in MSTA .Flow gradient differential histogram for the outline of a moving object combined with color histogram to reduce errors rate of MSTA tracking figure(2). 


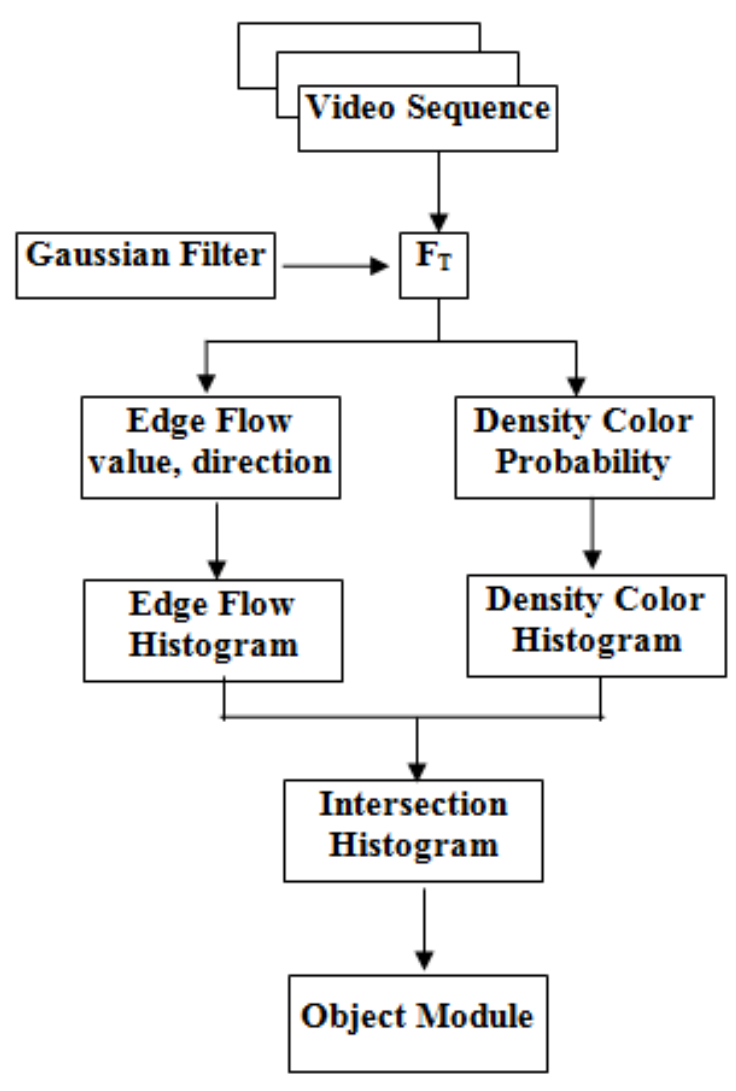

Fig2: Develop MSTA

Developed MSA using eq. (10) probability density object model $\mathrm{q}_{\mathrm{u}}$ by equation $10, \mathrm{u}=1 \ldots \ldots \mathrm{m}$ is

$\mathrm{q}_{\mathrm{u}}=\mathrm{C} \sum \mathrm{k}\left((|| \mathrm{xi}||)^{2} \delta\left(\mathrm{b}\left(\mathrm{x}_{\mathrm{i}}\right)-\mathrm{u}\right)\right.$

$\mathrm{C}$ is the normalization constant

$\mathrm{C}=\left(\sum \mathrm{k}(|| \mathrm{xi}|| 2)-1\right.$

$\sigma$ is the Kronecker delta function

$\delta(\mathrm{a})=1$ if $\mathrm{a}=0$ and $\delta(\mathrm{a})=0$ otherwise

The probability $\mathrm{p}$ of combined histogram $\mathrm{u}$ in the target candidate eq. 13

$\mathrm{P}_{\mathrm{u}}=\mathrm{c}_{\mathrm{h}} \sum \mathrm{k}\left(|| \mathrm{y}-\mathrm{y}_{\mathrm{i}} / \mathrm{h}||\right)^{2} \delta\left(\mathrm{b}\left(\mathrm{y}_{\mathrm{i}}\right)-\mathrm{u}\right)$

Use Mean Shift to estimate probability and target location. Track target candidate in video by matching probability of target model with candidate neighbor. Measure similarity between probability objects model $\mathrm{q}$ and target $\mathrm{p}$ at new location y use Bhattacharyya coefficient $\rho$. Let $\mathrm{z}$ denote estimated new target location near $y$, and probability does not change significantly eq. 14 .

$\rho(\mathrm{P}(\mathrm{z}), \mathrm{q})=1 / 2 \sum\left(\mathrm{p}_{\mathrm{u}}(\mathrm{y}) \mathrm{q}_{\mathrm{u}}\right)^{0.5}+\left(\mathrm{c}_{\mathrm{h}} / 2 \sum \mathrm{w}_{\mathrm{i}}\left(|| \mathrm{z}-\mathrm{y}_{\mathrm{i}} / \mathrm{h}||\right)^{2}\right)$

Where weight $\mathrm{w}_{\mathrm{i}}$ in eq. 15 equal to

$\left.\mathrm{w}_{\mathrm{i}}=\sum \delta\left(\mathrm{y}_{\mathrm{i}}\right)-\mathrm{u}\right)\left(\mathrm{q}_{\mathrm{u}} / \mathrm{p}_{\mathrm{u}}\left(\mathrm{y}_{0}\right)\right) \delta\left[\mathrm{b}\left(\mathrm{x}_{\mathrm{i}}\right)-\mathrm{u}\right)$

$\mathrm{z}$ new location of candidate target equation(16)

$\mathrm{Z}=\left(\sum\left[\mathrm{w}_{\mathrm{i}} \mathrm{g}\left(\left|\left(\mathrm{y}-\mathrm{y}_{\mathrm{i}}\right) / \mathrm{h}\right|\right)^{2} \mathrm{y}_{\mathrm{i}}\right] /\left(\sum\left[\mathrm{w}_{\mathrm{i}} \mathrm{g}\left(\left|\left(\mathrm{y}-\mathrm{y}_{\mathrm{i}}\right) / \mathrm{h}\right|\right)^{2}\right.\right.\right.$

Mean Shift Tracking Algorithm step:

Step1: Calculate target model by equation (10).
Step2: Initialize $\mathrm{y}_{0}$ location of candidate target in previous frame.

Step3: Calculate candidate model by equation (13) in current frame. a window of $y_{i}$ pixels is related to $h$ size of window.

(4) Compute weights $\mathrm{w}_{\mathrm{i}}$ according to equation 15.

(5) Apply mean shift: Compute new location $\mathrm{z}$ of target candidate equation 16 .

(5) Compute $\{\mathrm{pu}(\mathrm{z})\}, \mathrm{u}=1, \ldots, \mathrm{m}$, and _(p(z), q) equation 13.

(6) While $\rho(\mathrm{p}(\mathrm{z}), \mathrm{q})<\rho(\mathrm{p}(\mathrm{y}), \mathrm{q})$, do $\mathrm{z} \leftarrow 1$ This step used to validate the target's new location.

To adapt to change of scale, can modify window size $h$ and let algorithm converge to $h$ that maximizes $\rho(p(y, q))$.

(7) If $z_{k}-y_{k}$ is small enough, stop.

Else,

set $\mathrm{y} \leftarrow \mathrm{z}$ and go to Step 1 .

\section{EXPERIMENTAL RESULTS}

Proposed enhancement on target module representation to reduce error rate in classical MSA .This section introduces results for MSTA that use combined color and gradient histogram to represent region of interest in MSA.

First apply Gaussian filter to smooth sequence frames and soble operator for edge detection. Flow calculate by gradient differentials in four direction separately (Ix x , Iyx ,Ixy, Iyy ) to calculate edge flow-direction histograms. Use larger spatial displacements for differencing 5 pixels $[2,0,0,0,-2]$ mask, and take spatial differencing steps along several different directions . The motivation is that moving object width is approximately the same as the cell size they can capture relative displacements of the objects border with respect to the background. The maximum response gets from the sum square difference in all 8 neighbors represent magnate Edge Flow figure(3)
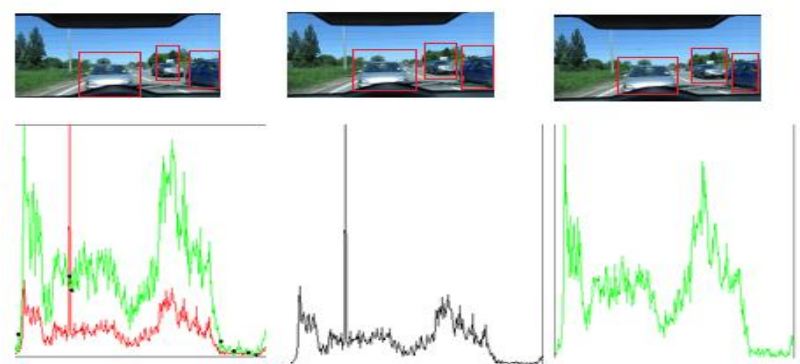

Fig3.a:multi objects
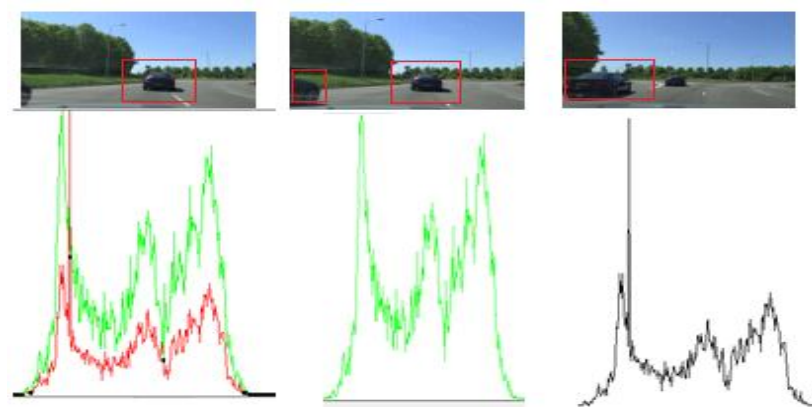

Fig3.b:new entry 

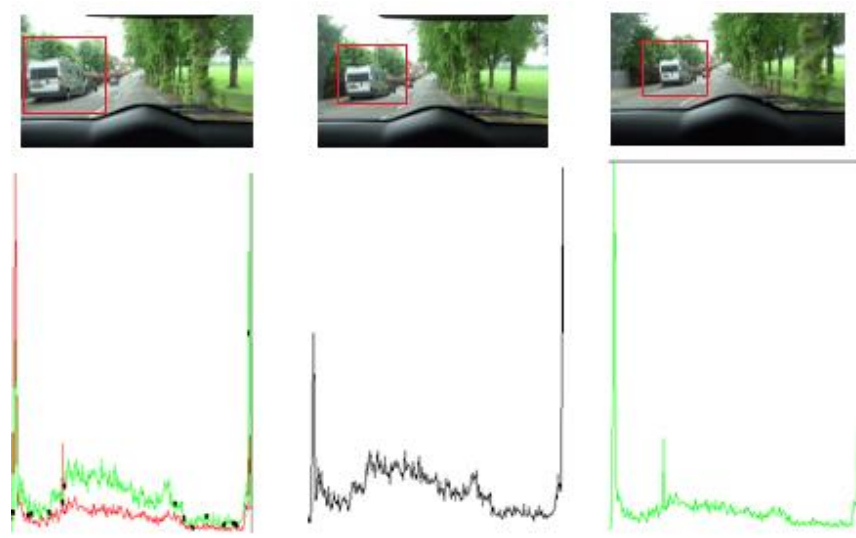

Fig3.c:stopping object
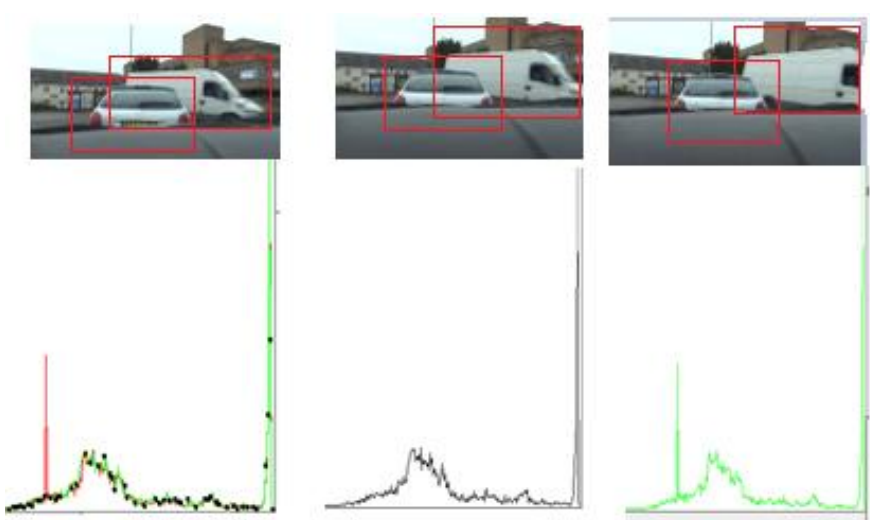

Fig3.d:partial occultation

New Target module has ability to track multi objects Fig.(3.a)show result of tracking multi objects in highway and second row show combined histogram, edge flow, color histogram. New entry to scene results show in fig(3.b).

Stopping object on scene capture from change edge flow fig(3.c).Occultation problem fig(4.d)in road and object moves slowly The correlation between qualitative precision results to initialize objects of interest given by the following measurements metric:

1-object Tracking Error(OTE):Euclidian distance between ground truth objects center and tracker object estimated by developed MSTA

Object Tracking Error $\left.(\mathrm{OTE})=\left[\left(\mathrm{x}-\mathrm{x}^{\mathrm{GT}}\right)^{2}+\right)+\left(\mathrm{y}-\mathrm{y}^{\mathrm{GT}}\right)^{2}\right]^{0.5}$

Where $(\mathrm{x}, \mathrm{y})$ object center estimated by developed MSTA, MSA,CBWH and (x GT, y GT) ground truth object center. compare with MSA and CBWH algorithm table(1) show the results of computation.

\section{Table1.Object Tracking Error}

\begin{tabular}{|c|c|c|c|c|l|}
\hline Algorithms & Video11 & Video44 & Video88 & Video99 & Car DB \\
\hline MSA & 2.745 & 2.828 & 2.645 & 2.645 & 2.755 \\
\hline CBWH & 2.45 & 2.449 & 2.448 & 2.448 & 2.446 \\
\hline $\begin{array}{c}\text { Developed } \\
\text { MSTA }\end{array}$ & 1.32 & 1.36 & 1.37 & 1.33 & 1.31 \\
\hline
\end{tabular}

Initialize geometric description of the object manually in MSA while $\mathrm{CBWH}$ initialized tracker target based on intensity both previous techniques work with assumption of constant object speed this assumption generate error increased along time

Accumulator Error $=\left[\left(\mathrm{x}-\mathrm{x}_{20}\right)^{2}+\left(\mathrm{y}-\mathrm{y}_{20}\right)^{2}\right]^{0.5}$

Where(x, y)estimated object center of first frame by developed MSTA,MSA,CBWH and (x20, y20) estimated object center for frame twenty. compare with MSA and CBWH algorithm table(2) show the results of computation.

\section{Table2.Accumulator Error}

\begin{tabular}{|c|c|c|c|c|}
\hline $\begin{array}{c}\text { Video } \\
\text { Name }\end{array}$ & $\begin{array}{c}\text { Object } \\
\text { Number }\end{array}$ & MSA & CBWH & $\begin{array}{c}\text { Developed } \\
\text { MSTA }\end{array}$ \\
\hline \multirow{2}{*}{ Video11 } & object 1 & 8.235 & 5.124 & 3.618 \\
\cline { 2 - 5 } & object 2 & 8.159 & 5.303 & 3.609 \\
\hline \multirow{2}{*}{ Video44 } & object 1 & 8.484 & 4.898 & 2.970 \\
\cline { 2 - 5 } & object 2 & 8.419 & 4.864 & 3.018 \\
\hline \multirow{3}{*}{ Video88 } & object1 & 7.931 & 4.896 & 2.774 \\
\cline { 2 - 5 } & object 2 & 7.972 & 4.889 & 2.965 \\
\cline { 2 - 5 } & object 3 & 7.989 & 4.907 & 3.125 \\
\hline Video99 & object 1 & 8.417 & 4.875 & 2.659 \\
\hline
\end{tabular}

Accurate Ratio (AR) measured errors between total ground truth points tracker object and total true estimated tracker object points. table(2) show Accurate Ratio measurement results.

Accurate $\operatorname{Ratio}(\mathrm{AR})=($ ground truth object points $) /($ true positives for tracked object).

Table3.Accurate Ratio

\begin{tabular}{|c|c|c|c|c|l|}
\hline Algorithms & Video11 & Vide044 & Video88 & Video99 & Car DB \\
\hline MSTA & 2.983 & 3.011 & 2.995 & 2.871 & 2.859 \\
\hline CBWH & 2.692 & 2.773 & 2.758 & 2.596 & 2.599 \\
\hline $\begin{array}{c}\text { Developed } \\
\text { MSTA }\end{array}$ & 1.121 & 1.2496 & 1.155 & 1.09 & 1.105 \\
\hline
\end{tabular}

Developed MSTA Accurate Ratio is steady on range between(0.004)while MSA(0.03) and $\mathrm{CBWH}(0.02)$ figure(4). 

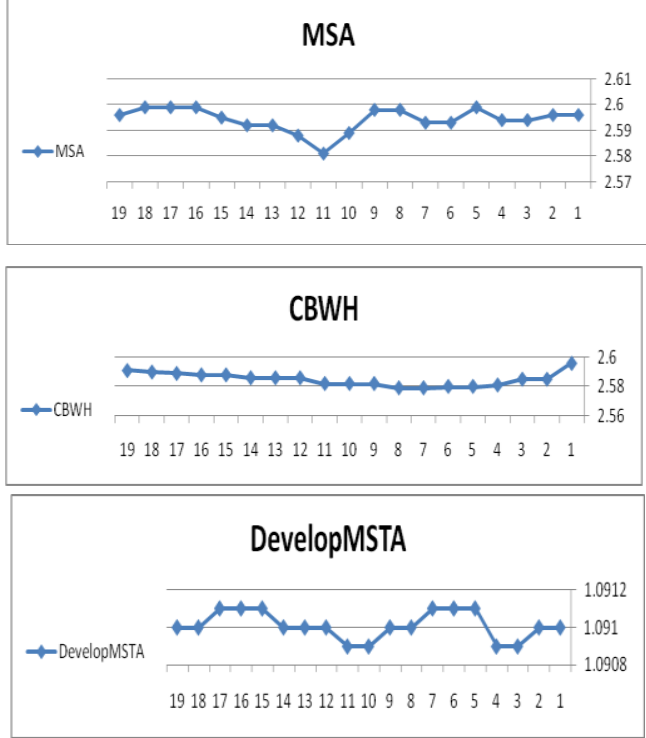

Fig4: Accurate Ratio

Our experiments show that using combined histogram to represent object model in MSTA reduces false tracking rate by a factor of more than 3 at $8 \%$ miss rate. In fact, aimed at improving object representation appears to enhance the tracker performance. Our method is also has the same MSA processing time, running 15 frame in 1 second on resolution $360 \times 240$

\section{CONCLUSION}

Image content is transformed into local feature coordinates that are invariant to: scale, rotation, viewpoint changes and illumination changes .This paper presents a development for tracking moving object by MSA .Our algorithm combines features extracted from a single frame of a video sequence with motion feature extracted from edge flow or spatiotemporal derivatives against the three sequence frame. Motion feature based on edge flow used with no prior knowledge about motion nature. Firstly start by estimating the motion from sequence frames using gradient differentials technique in (Ixx , Ixy , Iyx, Iyy ) separately. Secondly perform MSA using combined histogram for the color and maximum response to Edge Flow Histogram. We tested the developed method on real sequences frames. Experiment results are very promising to improved MSA. Combining motion and color histogram reduces the tracking error rate and handle occultation, new entry objects and multi objects.

\section{REFRENCES}

[1] B. Karasulu and S. Korukoglu, "Performance evaluation software moving object detection and tracking in videos", SpringerBriefs 7 in Computer Science, DOI: 10.1007/978-1-4614-6534-8_2013,

[2] N. Magoch T. \& M.M.Kuber, "A Comparative analysis of kernel-based target tracking methods using different colour feature based target models",Department of Computer Engineering, Defence Institute of Advanced Technology, Pune, India, International Journal of Computer Science and Informatics ISSN (PRINT): 2231 -5292 , Vol-1, Iss-4, 2012.

[3] S Ilić,Technischen,"Tracking and detection in computer vision Feature descriptors", University München Winter Semester 2009/2010.

[4] A. Yilmaz ,"Object Tracking A Survey”, Ohio State University,Omar Javed,ObjectVideo, Inc.and Mubarak Shah,University of Central Florida , ACM Computing Surveys, Vol. 38, No. 4, Article 13, Publication date: December 2006.

[5] M. Proesmans, L. Van Gool, E. Pauwels, and A Oosterlinck. "Determination of optical flow and its discontinuities using non-linear diffusion". In Proceedings of the 3rd European,Conference on Computer Vision, Stockholm, Sweden, volume 2, pages 295-304, 1994.

[6] D., Huang, Q., Jiang, S., Yao, H., Gao, W.” Mean-shift blob tracking with adaptive feature selection and scale adaptation". In International Conference Image Processing (2007).

[7] Q. Luo · X. Kong · G. Zeng, "Human action detection via boosted local motion histograms", Jianping Fan Received: 3 December 2007 , Accepted: 8 September 2008 @ Springer-Verlag 2008

[8] A. Yilmaz, M. Shah," Recognizing human actions in videos acquired by uncalibrated moving cameras". IEEE Int. Conf. Computer. Vis.1, 150-157 (2005)

[9] S.Kahlouche, O.Djekoune, D.Djebrouni, D.Meriche "Segmentation by motion based on optical flow histogram", Centre de Développement des Technologies Avancées CDTA,Houch Oukil, BP 17, Baba Hassen Alger - Algérie.

[10] C. Fennema and W. Thompson, "Velocity determination in scenes containing several moving objects", Computer Graphics and Image Processing,9 (1979), pp.301-315.

[11] R. Paquin and E. Dubois, « A spatio-temporal gradient method for estimating the displacement field in time varying imagery », Comp. Vis.,Graphics and Image Processing,Vol.21, pp.205-221,1983

[12] W. ,James ,R. Davis ,"Recognizing movement using motion histograms",MIT Media Laboratory, 20Ames Steet, Cambridge,MA Media Laboratory, Perceptual Computing Section Technical Report ,April.

[13] N. Dalal and B. Triggs. Histograms of oriented gradients for human detection. In Proceedings, of the Conference on Computer Vision and Pattern Recognition, San Diego, California,USA, pages 886-893, 2005 